\title{
Onset of exciton-exciton annihilation in single-layer black phosphorus
}

\author{
A. Surrente,${ }^{1}$ A. A. Mitioglu, ${ }^{1,2, *}$ K. Galkowski, ${ }^{1}$ L. Klopotowski, ${ }^{3}$ W. Tabis,${ }^{1,4}$ B. Vignolle, ${ }^{1}$ D. K. Maude, ${ }^{1}$ and P. Plochocka ${ }^{1, \dagger}$ \\ ${ }^{1}$ Laboratoire National des Champs Magnétiques Intenses, UPR 3228, CNRS-UGA-UPS-INSA, Grenoble and Toulouse, France \\ ${ }^{2}$ Institute of Applied Physics, Academiei Str. 5, Chisinau, MD-2028, Republic of Moldova \\ ${ }^{3}$ Institute of Physics, Polish Academy of Sciences, al. Lotników 32/46, 02-668 Warsaw, Poland \\ ${ }^{4}$ AGH University of Science and Technology, Faculty of Physics and Applied Computer Science, Al. Mickiewicza 30, 30-059 Krakow, Poland
}

(Received 18 May 2016; revised manuscript received 21 July 2016; published 18 August 2016)

\begin{abstract}
The exciton dynamics in monolayer black phosphorus is investigated over a very wide range of photoexcited exciton densities using time resolved photoluminescence. At low excitation densities, the exciton dynamics is successfully described in terms of a double exponential decay. With increasing exciton population, a fast, nonexponential component develops as exciton-exciton annihilation takes over as the dominant recombination mechanism under high excitation conditions. Our results identify an upper limit for the injection density, after which exciton-exciton annihilation reduces the quantum yield, which will significantly impact the performance of light emitting devices based on single-layer black phosphorus.
\end{abstract}

DOI: 10.1103/PhysRevB.94.075425

\section{INTRODUCTION}

The reduced dimensionality of atomically thin twodimensional (2D) semiconductors enhances many-body interactions, which influence strongly the carrier dynamics at high carrier concentrations. The large exciton binding energy in these layered materials [1-4] facilitates the observation of such many-body interactions in the exciton physics. In particular, exciton-exciton annihilation is a scattering mechanism in which one exciton recombines nonradiatively, transferring its energy and momentum to another exciton, which subsequently relaxes to lower energy states, losing the energy initially gained, through electron-phonon interactions. This process, which can be viewed as the exciton counterpart of Auger recombination for free carriers, has been demonstrated to be a very efficient nonradiative recombination channel (particularly at high injection levels) in low-dimensional systems such as carbon nanotubes [5-8], graphene nanoribbons [9], colloidal quantum dots and nanorods [10,11], organic semiconductors $[12,13]$, and monolayer transition metals dichalcogenides (TMDs) [14-18]. Other than the understanding of fundamental exciton dynamics, the interest of exciton-exciton annihilation resides in its significant technological impact, since it provides an upper limit for the carrier density under different excitation conditions. Exciton-exciton annihilation is therefore expected to have a significant influence on the efficiency of light emitting devices, such as semiconductor optical amplifiers and semiconductor lasers. It is also of interest in view of the production of highly efficient photovoltaic devices based on 2D materials. One of the factors limiting the efficiency of conventional solar cells is represented by the excess kinetic energy, dissipated as heat, of the hot carriers generated by the absorption of a photon with an energy well above the band gap. Efficient exciton-exciton annihilation might also imply

\footnotetext{
*Present address: High Field Magnet Laboratory (HFML-EMFL), Institute for Molecules and Materials, Radboud University, Toernooiveld 7, 6525 ED Nijmegen, The Netherlands.

†paulina.plochocka@lncmi.cnrs.fr
}

an efficient multiple exciton generation [19-21] (i.e., timereversed exciton-exciton annihilation) which could potentially lead to the harvesting of this excess energy, significantly reducing the influence of this loss channel.

Black phosphorus is a recent member of the rapidly expanding family of atomically thin 2D semiconductors obtained by mechanical exfoliation [22,23]. Its electronic properties combine the advantages of graphene in terms of carrier mobility with the direct band gap observed in monolayer TMDs. This results in an on/off ratio for black phosphorus field effect transistors of the order of $10^{5}$ [22,24,25]. The band gap of black phosphorus can be tuned from its bulk value $(0.3 \mathrm{eV})$ to the visible part of the spectrum by decreasing the number of layers [3,22]. As in TMDs, the strong carrier confinement and the decreased Coulomb screening due to the reduced dimensionality lead to an optical response of monolayer black phosphorus dominated by excitonic effects (the theoretical exciton binding energy is $0.8 \mathrm{eV}$ in vacuum [3] and $0.38 \mathrm{eV}$ on a $\mathrm{Si} / \mathrm{SiO}_{2}$ substrate [4]). The neutral exciton was reported to exhibit a photoluminescence (PL) emission energy of $\lesssim 2 \mathrm{eV}$ in monolayer samples [25-28]. Such properties make atomically thin black phosphorus an extremely promising material in electronics, for example to manufacture field effect transistors [22,24,25,29], and in optoelectronics with potential applications as photodetectors [30], or photon polarizers $[3,22,29,31,32]$. The responsivity of black phosphorus based photodetectors has been shown to decrease with increasing excitation power [30]. Although this effect has been attributed to a decrease of the available carriers created under high excitation conditions [30], the specific mechanism (saturation of trap states or Auger-like recombination) has yet to be unequivocally identified. As atomically thin black phosphorus has been successfully isolated only recently, very little is currently known about the carrier dynamics. Using pump-probe measurements, the evolution of exciton dynamics has been investigated, focusing on the in-plane anisotropy [33-35]. Most experiments have been performed at room temperature, and on thick samples [33], where the many-body effects are detrimentally weakened when compared to what is expected at low temperature on single atomic layers. One study 
reports the temporal evolution of the PL of single-layer black phosphorus, investigated over a limited range of excitation powers, always in the low excitation regime, with a single exponential PL decay [27].

In this paper, we present power dependent time-resolved micro-PL $(\mu \mathrm{PL})$ performed at low temperature. The pump fluence employed spans over almost four orders of magnitude, thereby providing access to the exciton dynamics in very different excitation regimes. We show that at low excitation densities the exciton dynamics in monolayer black phosphorus can be described using a double exponential decay. The first, shorter decay time, of the order of tens of picoseconds, is attributed to defect-mediated processes [36,37]. The longer decay time, of the order of a few hundreds of picoseconds, is a signature of the excitonic radiative recombination [36]. In the high fluence regime, both decay times decrease, pointing to the onset of many-body processes. Finally, at a yet higher exciton population, a fast, nonexponential decay component develops. Under this condition, the observed temporal dependence is best described by a bimolecular model involving exciton-exciton annihilation.

\section{EXPERIMENTAL DETAILS}

The bulk black phosphorus has been purchased from Smart Elements (99.998\% nominal purity). The mechanical exfoliation of the flakes was performed in a protected atmosphere (glove box filled with $\mathrm{Ar}$ gas, $<1 \mathrm{ppm}_{2},<1 \mathrm{ppm}_{2} \mathrm{O}$ ). The flakes were subsequently transferred onto a $\mathrm{Si}$ substrate capped with a 300-nm-thick layer of thermal $\mathrm{SiO}_{2}$. These samples were stored in vials, always in the glove box, and transferred to the He-flow cryostat used for the optical characterization, always under an Ar atmosphere. After mounting the samples, the cryostat was rapidly pumped to a pressure below $1 \times 10^{-4}$ mbar, minimizing the exposure of the black phosphorus to air. The single-layer character of the samples was verified by Raman measurements performed in situ on the same flake where the PL was investigated [28].

The steady-state $\mu \mathrm{PL}$ signal was excited with a $532 \mathrm{~nm}$ laser and the spectra were recorded using a spectrometer equipped with a liquid nitrogen cooled CCD camera. The time-resolved $\mu \mathrm{PL}$ signal was excited with the frequency doubled output of a tuneable optical parametric oscillator (OPO), synchronously pumped with a mode-locked Ti:sapphire laser. The typical temporal pulse width was $\simeq 300 \mathrm{fs}$, with a repetition rate of $80 \mathrm{mHz}$. The time-resolved PL signal was dispersed by an imagining spectrometer and detected using a synchroscan streak camera with the temporal resolution set to $\sim 10 \mathrm{ps}$. All the measurements presented here have been performed at $4.2 \mathrm{~K}$.

\section{RESULTS AND DISCUSSION}

The static excitonic response of the investigated flake was probed by time-integrated $\mu$ PL spectroscopy. A representative, low temperature $\mu \mathrm{PL}$ spectrum of the monolayer black phosphorus flake is shown in Fig. 1(a) (excitation power $P=30 \mu \mathrm{W})$. The spectrum consists of two distinct peaks, resulting from the recombination of the neutral $(X)$ and charged $(T)$ exciton.

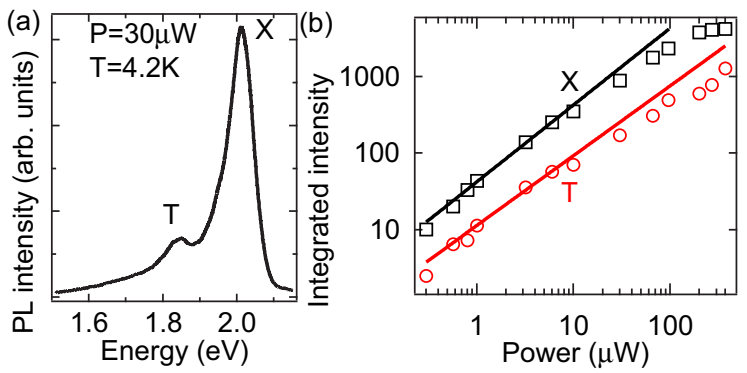

FIG. 1. (a) Time integrated $\mu$ PL spectrum of monolayer black phosphorus. (b) Integrated intensity of the neutral $(X)$ and charged $(T)$ exciton versus power. The solid lines represent fits to a power law.

In Fig. 1(b), we show the integrated intensity of the peaks versus excitation power. We fitted the integrated intensity $I$ to the power law $I \propto P^{n}$, obtaining $n=1.00 \pm 0.02$ for the neutral exciton and $n=0.91 \pm 0.05$ for the charged exciton for $P \leqslant 10 \mu \mathrm{W}$. This confirms that in both cases the recombination process involves a single electron-hole pair [28]. At higher excitation power, the integrated intensity of this flake shows a sublinear increase as a function of the excitation power, with a clear saturation of the intensity of the neutral exciton for $P \geqslant 200 \mu \mathrm{W}$. Considering that the band gap of black phosphorus (and hence its emission energy) is known to blueshift with increasing temperature [28,38,39], we can rule out laser-induced heating effects even at the highest excitation intensity, as no shift of the PL emission energy is observed for this sample. This, along with the constant line shape [28] (pointing to the absence of biexciton recombination), the saturation of the PL intensity at high excitation power, and the 2D excitonic character of the emission of black phosphorus, suggests that other nonlinear processes, namely exciton-exciton annihilation, play an important role [13-18].

To elucidate the origin of the observed nonlinear behavior of the black phosphorus PL, we performed systematic measurements of time-resolved $\mu \mathrm{PL}$ at a varying excitation density (see Fig. 2). For reference, in Fig. 2 we also show the temporal evolution of the excitation pulse, which represents the lower limit of the temporal resolution of our detection system (instrument response function, IRF, with a full width at half maximum of $\sim 10 \mathrm{ps}$, as extracted from a Gaussian fit). We note that even at the lowest pump fluence, the time decay of the neutral exciton is characterized by two different slopes, indicating the presence of at least two decay components. Moreover, with increasing fluence, the fast decay component becomes more significant, as attested by the prominent peak of the time-resolved traces at short delay times, whereas the slower decay components tends to decrease, with a corresponding smaller slope observed at larger delay times at high power.

To verify whether the time decay at low pump fluence (or equivalently at low exciton density) could be described in terms of only radiative recombination, we have fitted the PL intensity $I(t)$ to a single exponential decay convoluted with a Gaussian IRF, $I(t)=\int_{-\infty}^{t} \operatorname{IRF}\left(t^{\prime}\right) \mathrm{e}^{-\left(t-t^{\prime}\right) / \tau} \mathrm{d} t^{\prime}$. In the left column of Fig. 3 we present the results of these fits. Except for the lowest fluence, the single exponential model is 


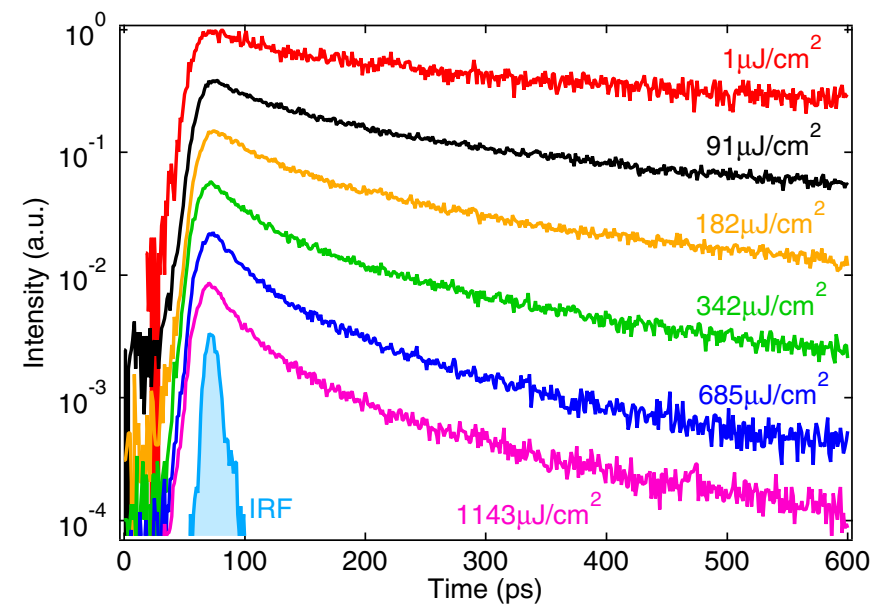

FIG. 2. Time decay of the neutral excitonic transition at various pump fluences. The instrument response function (IRF) is shown for reference. The curves are vertically offset for clarity.

unable to fit satisfactorily the data, showing a large difference with respect to the measured decay especially at very short and at long $t$. This demonstrates that a second relaxation mechanism (other than electron-hole recombination) has to play a role in the investigated sample. A wide range of defects or impurities are expected to influence the electronic properties of mechanically exfoliated black phosphorus monolayers [40]. Such defects could induce trap states for the excitons, which are periodically populated and depopulated. The combination of this relaxation path with the electron-hole recombination can be accounted for by a double exponential decay, in which the PL intensity is described by $I(t)=$ $\int_{-\infty}^{t} \operatorname{IRF}\left(t^{\prime}\right)\left[\mathrm{e}^{-\left(t-t^{\prime}\right) / \tau_{\mathrm{d}}}+a \mathrm{e}^{-\left(t-t^{\prime}\right) / \tau}\right] \mathrm{d} t^{\prime}$, where $a$ denotes the ratio between the two exponential components, and $\tau_{\mathrm{d}}$ and $\tau$ are decay times due to the defect related path and radiative

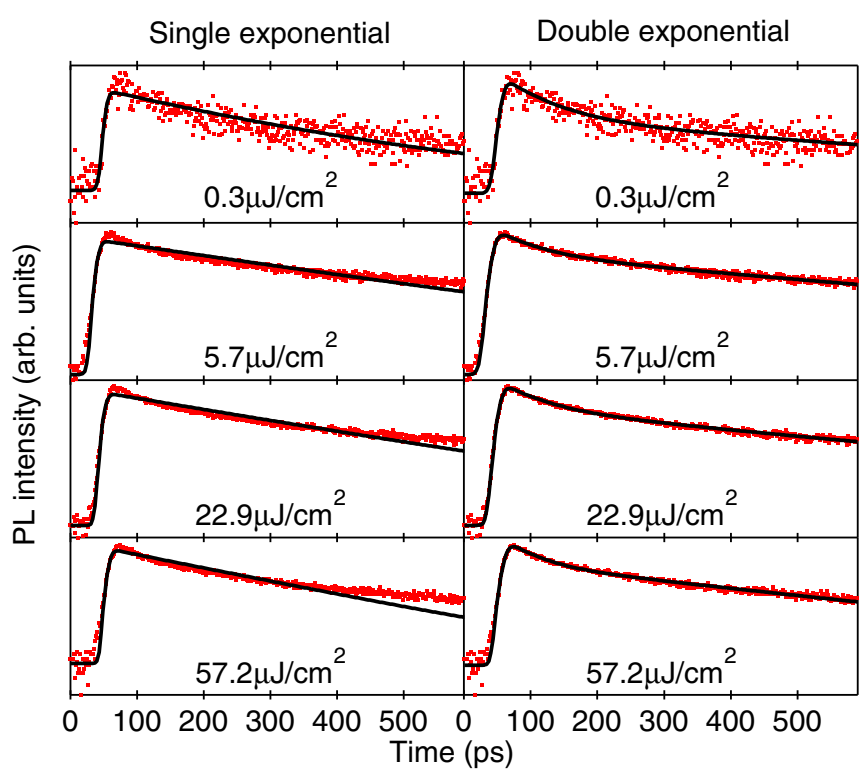

FIG. 3. Time-resolved $\mu \mathrm{PL}$ measurements of neutral exciton (dotted curves) and fits to single exponential and double exponential models (solid lines). The pump fluences are indicated in each panel.

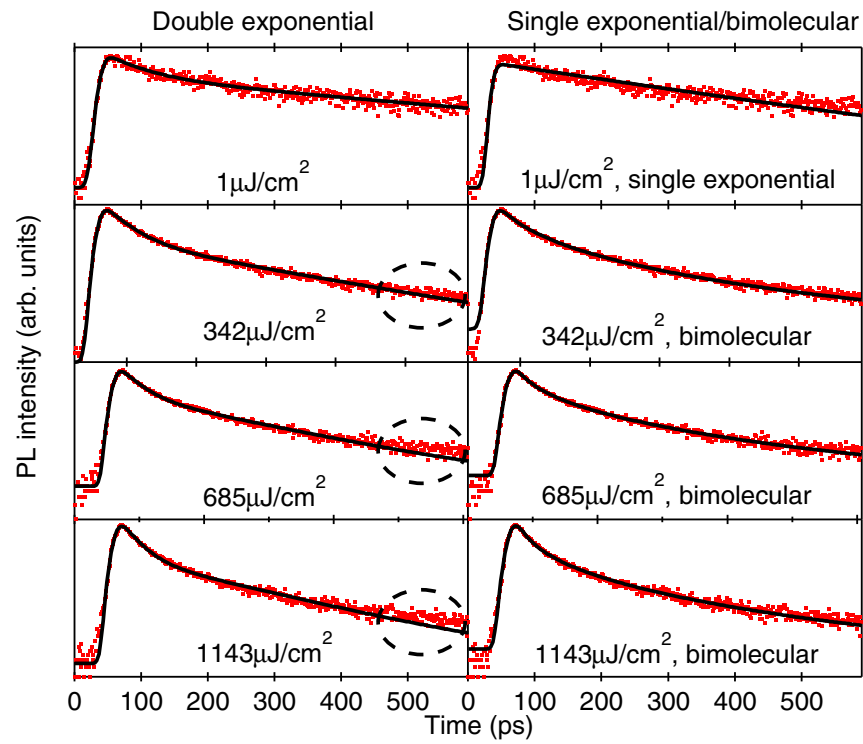

FIG. 4. Time decay of neutral exciton (dotted curves) and fits to single exponential, double exponential, and bimolecular models (solid lines). The dashed ellipses highlight the delay times at which the double exponential mode is unable to fit the data.

recombination, respectively. Using this model to describe the data leads to a considerably improved quality of the fits, at least at low and intermediate pump fluence. This is demonstrated by the fits presented in the right column of Fig. 3, which capture the time decay of the PL signal significantly better, suggesting that defect-mediated trapping relaxation plays an important role in the mechanically exfoliated black phosphorus monolayers. We attribute the fast decay to trapping or defect-assisted recombination mechanisms, as previously reported in TMDs [36,37], while the slow decay reflects the exciton radiative recombination [36]. Our results at low fluence are not surprising, since similar, multiexponential decays have already been reported for black phosphorus [33-35]. Fitting with the double exponential model all the data measured at a different excitation power gives values of $\tau_{\mathrm{d}}$ of tens of picoseconds and values of $\tau$ of hundreds of picoseconds [see Fig. 5(a) for the exact values].

The time-resolved $\mu \mathrm{PL}$ measured at high fluence is shown in Fig. 4. Obtaining the best fit with a double exponential model (see left column of Fig. 4) requires a varying ratio $a$ between components with increasing excitation, as summarized in Fig. 5(b). We note that if the exciton dynamics was solely dominated by the two mechanisms mentioned above, the saturation of the exciton traps at very high fluence would lead to an increasing weight of the radiative recombination component $a$ [15]. Here, at the lowest pump fluences $a$ increases slightly and then remains essentially constant up to $\sim 20 \mu \mathrm{J} \mathrm{cm}{ }^{2}$, indicating that the trap states are being gradually filled, although probably not up to the full saturation. At higher pump fluence the opposite trend is experimentally observed, with the amplitude ratio decreasing significantly with increasing fluence [see Fig. 5(b)]. This, along with variation of the extracted decay times $\tau$ and $\tau_{\mathrm{d}}$ as a function of the pump fluence [see Fig. 5(a)], suggests the occurrence of nonlinear processes coming into play when the exciton 

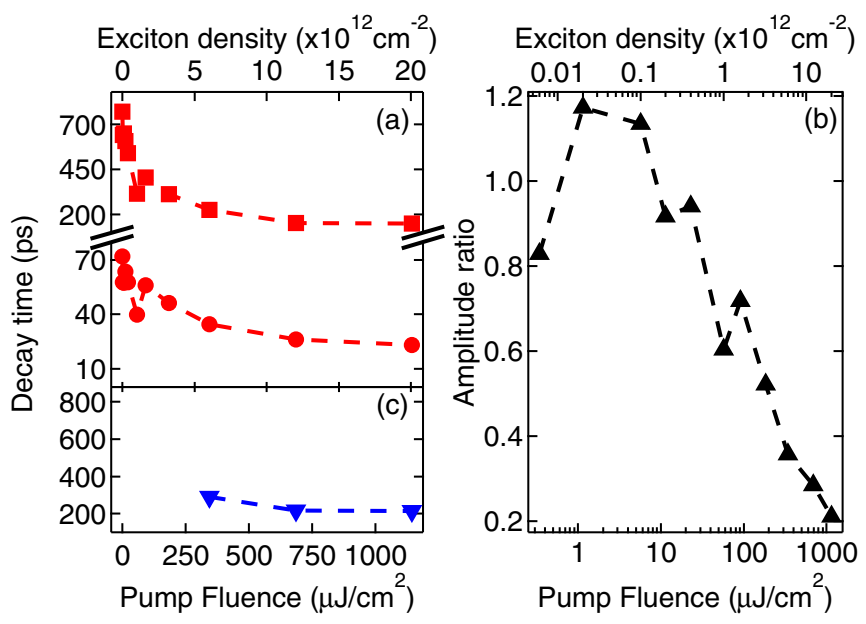

FIG. 5. (a) Decay times and (b) ratio between the two exponential components versus pump fluence and initial exciton density, double exponential model. (c) Decay time as a function of pump fluence and initial exciton density, bimolecular model. The dashed lines are a guide to the eye.

population is sufficiently large. This is corroborated by the failure of the double exponential model to fit the exciton dynamics measured at pump fluence larger than $\geqslant 342 \mu \mathrm{J} \mathrm{cm}^{2}$, as can be seen, for example, in the fit of the high fluence time-resolved $\mu \mathrm{PL}$ displayed in the left column of Fig. 4 (note in particular the discrepancy occurring at large delay times). Such an excitation level corresponds to the excitation regime at which the integrated intensity saturates, as shown in Fig. 1(b). The observation of a sublinear dependence of the integrated intensity on the excitation power and of the appearance of fast, nonexponential exciton dynamics at the same excitation level has already been interpreted by referring to exciton-exciton annihilation processes [17].

We now focus on the exciton dynamics in the nonlinear regime. At high pump fluence, the fast decay component becomes more prominent, as evidenced by the faster initial decay (see Fig. 2). We rule out the contribution related to the formation of biexcitonic complexes, which might possibly be responsible for an increasing fast decay component, as no additional low energy peaks corresponding to biexcitonic recombination are observed at high power [28]. Instead, we propose exciton-exciton annihilation, already observed in TMDs [14-18], as the recombination mechanism responsible for the observed nonlinear exciton dynamics. In this framework, the rate equation for the exciton population $n(t)$ can be written:

$$
\frac{\partial n}{\partial t}=-\frac{n}{\tau}-\gamma n^{2}
$$

where $\tau$ is the exciton lifetime, and $\gamma$ is the annihilation rate. At high fluences, the quadratic (bimolecular) term leads to a rapid decay which dominates over the defect channel which can then be neglected. We would like to stress that the bimolecular term is too small to explain the fast decay at low fluences, for which the double exponential model, which includes the defect channel, is required.

Assuming that $\gamma$ is independent of time, and in the regime of very short excitation pulse $\left(\tau_{\text {pulse }} \ll \tau\right)$, the PL temporal dependence $I_{\mathrm{bm}}(t)$ in the bimolecular model can be written as $[12,13,17]$

$$
I_{\mathrm{bm}}(t)=\frac{I_{\mathrm{in}} \mathrm{e}^{-t / \tau}}{1+\gamma \tau n(0)\left(1-\mathrm{e}^{-t / \tau}\right)},
$$

where $I_{\text {in }}$ denotes the pump intensity and $n(0)$ represents the initial exciton density, estimated [41] by taking into account the Gaussian profile of the excitation spot, its size $(\sim 1 \mu \mathrm{m})$, the excitation wavelength $(526 \mathrm{~nm})$ and the absorbance of monolayer black phosphorus of $2.8 \%$ per layer [4]. By convoluting $I_{\mathrm{bm}}(t)$ with the IRF, we have extracted the exciton lifetimes and the annihilation rates for pump fluences larger than $342 \mu \mathrm{J} \mathrm{cm}^{2}$. We found a decay time $\tau \lesssim 280 \mathrm{ps}$ [see Fig. 5(c) for the detailed fluence dependence], similar to the $\tau$ obtained at low pump fluence in the double exponential model. All the high fluence decays could be fitted using an almost constant value of annihilation rate $\gamma=5.0 \pm 0.2 \times 10^{-3} \mathrm{~cm}^{2} / \mathrm{s}$. Therefore, if we restrict our analysis to the highest excitation conditions, we can successfully fit the exciton dynamics with a single set of parameters, suggesting that exciton-exciton annihilation becomes the dominant recombination mechanism in the investigated sample at an exciton concentration of $n(0) \sim$ $6.1 \times 10^{12} \mathrm{~cm}^{-2}$. The validity of the bimolecular model at very high pump fluence is further corroborated by the comparison of $\tau_{\mathrm{d}}$ with the effective, exciton-density-dependent fast time constant resulting from the bimolecular model $(\gamma n)^{-1}$. For fluences larger than $340 \mu \mathrm{J} \mathrm{cm}^{2}$, our fitting parameters show that $(\gamma n)^{-1}<\tau_{\mathrm{d}}$ (at the highest exciton densities by more than a factor of 2), demonstrating that at these excitation conditions the fast exciton dynamics is dominated by nonlinear processes, and allowing us to neglect the influence of defect-mediated recombination. The annihilation rate is related to the exciton diffusion constant $D$ and to the maximum distance $r_{\mathrm{a}}$ at which the annihilation process occurs through $\gamma=4 \pi D r_{\mathrm{a}}$ [42]. The substrate could affect the measured annihilation rate either by reducing the binding energy [4], which might lead to a decrease of $r_{\mathrm{a}}$, or through a reduced mobility [43], which can influence the exciton diffusion constant. Moreover, the presence of defects in the mechanically exfoliated flakes introduces an additional recombination pathway in competition with the exciton-exciton annihilation, partially smearing its effects [16]. This suggests that in the case of a better crystalline quality of monolayer black phosphorus, exciton-exciton annihilation could occur at lower exciton densities.

The recently reported annihilation rates of monolayer TMDs range from $\sim 0.04-0.1 \mathrm{~cm}^{2} / \mathrm{s}$ for $\mathrm{MoS}_{2}[15,16]$ to $\sim 0.3-0.4 \mathrm{~cm}^{2} / \mathrm{s}$ for $\mathrm{WSe}_{2}$ [18], $\mathrm{WS}_{2}$ [16,17], and $\mathrm{MoSe}_{2}$ [14], with a typical exciton density of the order of $10 \times 10^{10} \mathrm{~cm}^{-2}$ [14-18]. The lower annihilation rate of monolayer black phosphorus of $5.0 \pm 0.2 \times 10^{-3} \mathrm{~cm}^{2} / \mathrm{s}$ obtained from our measurements is thus consistent with the larger exciton density that has to be injected to observe a significant exciton-exciton annihilation.

\section{CONCLUSIONS}

In summary, we have presented a detailed study of the power dependence of the neutral exciton dynamics in monolayer black phosphorus investigated using time-resolved $\mu \mathrm{PL}$ measurements. Varying the pump fluence over almost four 
orders of magnitudes allowed us to probe the exciton dynamics at strongly different photoexcited exciton densities. At low pump fluence, the measured PL decay can be satisfactorily fitted with a double exponential model. In this framework, the fast decay is assigned to defect-mediated charging and decharging mechanisms, while the long-lived component is attributed to the radiative recombination of the excitons. As the pump fluence was increased, the ratio between the two decay components varies, with the fast decay component taking over in the high fluence regime, which is completely inconsistent with the expected behavior in the case of a saturation of the trapping defects. The observation of this fast, nonexponential decay component can be explained in terms of exciton-exciton annihilation. Our results allow us to identify an upper limit for the density of injected carriers above which the exciton-exciton annihilation regime completely dominates.
The threshold exciton density is $\sim 6.1 \times 10^{12} \mathrm{~cm}^{-2}$. Above this density a single set of fitting parameters can be used to fit the PL decay. The demonstrated domination of exciton-exciton annihilation has important implications for the optimization of the quantum yield of advanced light emitting devices and for the responsivity of photodetectors based on monolayer black phosphorus.

\section{ACKNOWLEDGMENTS}

The authors gratefully acknowledge Geert Rikken for providing the bulk black phosphorus. A.A.M. acknowledges financial support from the French foreign ministry. This work was partially supported by ANR JCJC project milliPICS, the Région Midi-Pyrénées under Contract MESR 1305303, and the BLAPHENE project under IDEX program Emergence.
[1] K. He, N. Kumar, L. Zhao, Z. Wang, K. F. Mak, H. Zhao, and J. Shan, Phys. Rev. Lett. 113, 026803 (2014).

[2] H. M. Hill, A. F. Rigosi, C. Roquelet, A. Chernikov, T. C. Berkelbach, D. R. Reichman, M. S. Hybertsen, L. E. Brus, and T. F. Heinz, Nano Lett. 15, 2992 (2015).

[3] V. Tran, R. Soklaski, Y. Liang, and L. Yang, Phys. Rev. B 89, 235319 (2014).

[4] A. Castellanos-Gomez, L. Vicarelli, E. Prada, J. O. Island, K. L. Narasimha-Acharya, S. I. Blanter, D. J. Groenendijk, M. Buscema, G. A. Steele, J. V. Alvarez, H. W. Zandbergen, J. J. Palacios, and H. S. J. van der Zant, 2D Mater. 1, 025001 (2014).

[5] L. Lueer, S. Hoseinkhani, D. Polli, J. Crochet, T. Hertel, and G. Lanzani, Nat. Phys. 5, 54 (2009).

[6] F. Wang, G. Dukovic, E. Knoesel, L. E. Brus, and T. F. Heinz, Phys. Rev. B 70, 241403 (2004).

[7] L. Huang and T. D. Krauss, Phys. Rev. Lett. 96, 057407 (2006).

[8] Y.-Z. Ma, L. Valkunas, S. L. Dexheimer, S. M. Bachilo, and G. R. Fleming, Phys. Rev. Lett. 94, 157402 (2005).

[9] G. Soavi, S. Dal Conte, C. Manzoni, D. Viola, A. Narita, Y. Hu, X. Feng, U. Hohenester, E. Molinari, D. Prezzi, K. Müllen, and G. Cerullo, Nat. Commun. 7, 11010 (2016).

[10] V. I. Klimov, A. A. Mikhailovsky, D. W. McBranch, C. A. Leatherdale, and M. G. Bawendi, Science 287, 1011 (2000).

[11] H. Htoon, J. A. Hollingsworth, R. Dickerson, and V. I. Klimov, Phys. Rev. Lett. 91, 227401 (2003).

[12] P. E. Shaw, A. Ruseckas, and I. D. W. Samuel, Adv. Mater. 20, 3516 (2008).

[13] G. M. Akselrod, Y. R. Tischler, E. R. Young, D. G. Nocera, and V. Bulovic, Phys. Rev. B 82, 113106 (2010).

[14] N. Kumar, Q. Cui, F. Ceballos, D. He, Y. Wang, and H. Zhao, Phys. Rev. B 89, 125427 (2014).

[15] D. Sun, Y. Rao, G. A. Reider, G. Chen, Y. You, L. Brézin, A. R. Harutyunyan, and T. F. Heinz, Nano Lett. 14, 5625 (2014).

[16] Y. Yu, Y. Yu, C. Xu, A. Barrette, K. Gundogdu, and L. Cao, Phys. Rev. B 93, 201111 (2016).

[17] L. Yuan and L. Huang, Nanoscale 7, 7402 (2015).
[18] S. Mouri, Y. Miyauchi, M. Toh, W. Zhao, G. Eda, and K. Matsuda, Phys. Rev. B 90, 155449 (2014).

[19] R. D. Schaller and V. I. Klimov, Phys. Rev. Lett. 92, 186601 (2004).

[20] L. A. Padilha, J. T. Stewart, R. L. Sandberg, W. K. Bae, W.-K. Koh, J. M. Pietryga, and V. I. Klimov, Nano Lett. 13, 1092 (2013).

[21] N. J. L. K. Davis, M. L. Böhm, M. Tabachnyk, F. WisniveskyRocca-Rivarola, T. C. Jellicoe, C. Ducati, B. Ehrler, and N. C. Greenham, Nat. Commun. 6, 8259 (2015).

[22] X. Ling, H. Wang, S. Huang, F. Xia, and M. S. Dresselhaus, Proc. Natl. Acad. Sci. USA 112, 4523 (2015).

[23] A. Castellanos-Gomez, J. Phys. Chem. Lett. 6, 4280 (2015).

[24] L. Li, Y. Yu, G. J. Ye, Q. Ge, X. Ou, H. Wu, D. Feng, X. H. Chen, and Y. Zhang, Nat. Nanotechnol. 9, 372 (2014).

[25] H. Liu, A. T. Neal, Z. Zhu, Z. Luo, X. Xu, D. Tománek, and P. D. Ye, ACS Nano 8, 4033 (2014).

[26] X. Wang, A. M. Jones, K. L. Seyler, V. Tran, Y. Jia, H. Zhao, H. Wang, L. Yang, X. Xu, and F. Xia, Nat. Nanotechnol. 10, 517 (2015).

[27] J. Yang, R. Xu, J. Pei, Y. W. Myint, F. Wang, Z. Wang, S. Zhang, Z. Yu, and Y. Lu, Light: Science \& Applic. 4, e312 (2015).

[28] A. Surrente, A. A. Mitioglu, K. Galkowski, W. Tabis, D. K. Maude, and P. Plochocka, Phys. Rev. B 93, 121405(R) (2016).

[29] F. Xia, H. Wang, and Y. Jia, Nat. Commun. 5, 4458 (2014).

[30] M. Buscema, D. J. Groenendijk, S. I. Blanter, G. A. Steele, H. S. J. van der Zant, and A. Castellanos-Gomez, Nano Lett. 14, 3347 (2014).

[31] T. Low, A. S. Rodin, A. Carvalho, Y. Jiang, H. Wang, F. Xia, and A. H. Castro Neto, Phys. Rev. B 90, 075434 (2014).

[32] J. Qiao, X. Kong, Z.-X. Hu, F. Yang, and W. Ji, Nat. Commun. 5, 4475 (2014).

[33] S. Ge, C. Li, Z. Zhang, C. Zhang, Y. Zhang, J. Qiu, Q. Wang, J. Liu, S. Jia, J. Feng, and D. Sun, Nano Lett. 15, 4650 (2015).

[34] R. J. Suess, M. M. Jadidi, T. E. Murphy, and M. Mittendorff, Appl. Phys. Lett. 107, 081103 (2015).

[35] J. He, D. He, Y. Wang, Q. Cui, M. Z. Bellus, H.-Y. Chiu, and H. Zhao, ACS Nano 9, 6436 (2015).

[36] H. Shi, R. Yan, S. Bertolazzi, J. Brivio, B. Gao, A. Kis, D. Jena, H. G. Xing, and L. Huang, ACS Nano 7, 1072 (2013). 
[37] H. Wang, C. Zhang, and F. Rana, Nano Lett. 15, 339 (2014).

[38] D. Warschauer, J. Appl. Phys. 34, 1853 (1963).

[39] M. Baba, Y. Nakamura, K. Shibata, and A. Morita, Jpn. J. Appl. Phys. 30, L1178 (1991).

[40] S. Yuan, A. N. Rudenko, and M. I. Katsnelson, Phys. Rev. B 91, 115436 (2015).
[41] C. Jordan, J. F. Donegan, J. Hegarty, B. J. Roycroft, S. Taniguchi, T. Hino, E. Kato, N. Noguchi, and I. Ishibashi, Appl. Phys. Lett. 74, 3359 (1999).

[42] P. E. Shaw, A. Ruseckas, J. Peet, G. C. Bazan, and I. D. W. Samuel, Adv. Funct. Mater. 20, 155 (2010).

[43] N. Ma and D. Jena, Phys. Rev. X 4, 011043 (2014). 